\title{
Intrinsic resonant enhancement of light by nonlinear vacuum
}

\author{
Kazunori Shibata ${ }^{\mathrm{a}}$ \\ Institute of Laser Engineering, Osaka University, 2-6 Yamada-Oka, Suita, Osaka 565-0871, Japan
}

Received 30 July 2020 / Received in final form 17 September 2020 / Accepted 23 September 2020

Published online 15 October 2020

(C) The Author(s) 2020. This article is published with open access at Springerlink.com

\begin{abstract}
In nonlinear electromagnetism in vacuum, a classical electromagnetic wave itself can generate another wave. A classical field can become a source for a nonlinear correction via the polarization and magnetization of vacuum. We elucidate that a resonant generation is intrinsic to the theoretical structure of nonlinear electromagnetism. The resonance can take place when the phases, or the cycles of the source and the nonlinear correction match. We demonstrate two specific systems as examples. For a plane wave and constant fields, nonlinear corrective electromagnetic fields are resonantly enhanced with distance. It is shown in a stationary special solution. For more realistic system, we have considered the case of a standing wave in a cavity with an appropriate initial and boundary conditions. As a result, the corrections are resonantly enhanced with time. The resonance effect in the cavity is shown to be observed more effectively by combining a static magnetic flux density. We have evaluated the resonant effect using concrete parameters of current experiments. The demonstrated resonance can be combined with existing proposals to enable experimental detection of nonlinear optical effects of vacuum easier.
\end{abstract}

\section{Introduction}

Extended electromagnetic models have been considered [1-3]. These theories describe the electromagnetic nonlinearity of vacuum and have recently drawn attention across several fields, such as photon-photon scattering in quantum mechanics [4], radiation from stars in astrophysics [5-7], energy levels of hydrogen atom in atomic physics $[8,9]$, and high intensity laser physics [10-12], where uncharted range of strong electromagnetic fields should be properly treated. Several tests have been performed $[13,14]$ and many proposals are considered [15-21], however, an experimental observation of the nonlinear vacuum has remained a challenge.

Two reasons can account for the difficulty. One is that the nonlinearity requires extremely strong electromagnetic fields, e.g., the expected constraint given by the Schwinger limit $E_{\mathrm{sch}} \approx 1.32 \times 10^{18}(\mathrm{~N} / \mathrm{C})$ [3]. Another is that the nonlinear effects depend on electromagnetic fields through two Lorentz invariants [22], and not on energy density. For example, these invariants become zero in a plane wave regardless of the amplitude. It can easily occur that the energy density is large but the invariants are small. This theoretical structure has become a restriction on designing an efficient experimental setup.

In order to overcome the difficulty, we theoretically investigate nonlinear corrections that stem from classical electromagnetic fields. We show that, in a specific physical system, the nonlinear correction can increase resonantly and the resonance is intrinsic to nonlinear electromag-

\footnotetext{
${ }^{a}$ e-mail: shibata-ka@ile.osaka-u.ac.jp
}

netism. We also describe the reason why the resonance can be utilized for a feasible experiment of nonlinear vacuum.

\section{Notations}

For simplicity, we normalize the electromagnetic functions by the electric constant $\varepsilon_{0}$ and magnetic constant $\mu_{0}$. The electric field is multiplied by $\varepsilon_{0}^{1 / 2}$, the polarization of vacuum is divided by $\varepsilon_{0}^{1 / 2}$, the magnetic flux density is divided by $\mu_{0}^{1 / 2}$, and the magnetization of vacuum is multiplied by $\mu_{0}^{1 / 2}$. In addition, arguments of the functions are omitted when it is not confusing.

Using the electric field $\boldsymbol{E}$ and magnetic flux density $\boldsymbol{B}$, we introduce two Lorentz invariants by $F=E^{2}-B^{2}$ and $G=\boldsymbol{E} \cdot \boldsymbol{B}$. The nonlinear electromagnetic Lagrangian we consider herein is given by

$$
L=\frac{1}{2} F+C_{2,0} F^{2}+C_{0,2} G^{2}
$$

where $C_{2,0}$ and $C_{0,2}$ are nonlinear parameters [23]. Although this study is not limited to the Heisenberg-Euler model, we use the values of the model for several quantitative evaluations, i.e., $C_{2,0}^{(\mathrm{HE})}=1.665 \times 10^{-30}\left(\mathrm{~m}^{3} / \mathrm{J}\right)$ and $C_{0,2}^{(\mathrm{HE})}=7 C_{2,0}^{(\mathrm{HE})}[3]$.

The polarization and magnetization of vacuum are defined by

$$
\begin{aligned}
& \boldsymbol{P}=4 C_{2,0} F \boldsymbol{E}+2 C_{0,2} G \boldsymbol{B}, \\
& \boldsymbol{M}=-4 C_{2,0} F \boldsymbol{B}+2 C_{0,2} G \boldsymbol{E},
\end{aligned}
$$


respectively. Then, the nonlinear Maxwell's equations in this study are given by

$$
\begin{aligned}
& \nabla \cdot \boldsymbol{B}=0, \\
& \nabla \times \boldsymbol{E}+c^{-1} \partial_{t} \boldsymbol{B}=\mathbf{0}, \\
& \nabla \cdot \boldsymbol{E}=-\nabla \cdot \boldsymbol{P}, \\
& \nabla \times \boldsymbol{B}-c^{-1} \partial_{t} \boldsymbol{E}=c^{-1} \partial_{t} \boldsymbol{P}+\nabla \times \boldsymbol{M},
\end{aligned}
$$

where $c$ is the speed of light and $\partial_{t}$ expresses the partial differentiation with respect to time. If the right-hand sides of the third and fourth equations are replaced by zero, the above equations become classical Maxwell's equations.

A classical electromagnetic field that satisfies classical Maxwell's equations does not always satisfy nonlinear Maxwell's equations. In this case, the total electromagnetic field can be expressed by a sum of classical terms and a nonlinear corrective term such as $\boldsymbol{E}=\boldsymbol{E}_{c}+\boldsymbol{E}_{n}$ and $\boldsymbol{B}=\boldsymbol{B}_{c}+\boldsymbol{B}_{n}$, where the subscripts $c$ and $n$ exhibit the classical and nonlinear corrective terms, respectively. We would like to design a physical system where an effect of the nonlinear Lagrangian appears more strongly. For this purpose, the larger corrective terms are preferable.

In the present study, both $\boldsymbol{P}$ and $\boldsymbol{M}$ are cubic with respect to $\boldsymbol{E}$ and $\boldsymbol{B}$. Thus, considering the entire $\boldsymbol{P}$ and $\boldsymbol{M}$ is complicated. However, in many experimental situations, the classical terms are overwhelmingly larger than the corrective terms. Then, the main contributions to $\boldsymbol{P}$ and $\boldsymbol{M}$ will be given only by the classical terms. Let $F_{c}=E_{c}^{2}-B_{c}^{2}$ and $G_{c}=\boldsymbol{E}_{c} \cdot \boldsymbol{B}_{c}$, such contributions can be expressed by

$$
\begin{aligned}
& \boldsymbol{P}^{(0)}=4 C_{2,0} F_{c} \boldsymbol{E}_{c}+2 C_{0,2} G_{c} \boldsymbol{B}_{c}, \\
& \boldsymbol{M}^{(0)}=-4 C_{2,0} F_{c} \boldsymbol{B}_{c}+2 C_{0,2} G_{c} \boldsymbol{E}_{c},
\end{aligned}
$$

and $\boldsymbol{P} \approx \boldsymbol{P}^{(0)}$ and $\boldsymbol{M} \approx \boldsymbol{M}^{(0)}$ will hold, respectively. Then, the corresponding corrective terms of $\boldsymbol{E}_{n}$ and $\boldsymbol{B}_{n}$ will be the leading term in the nonlinear correction. We call them as the minimum corrective terms and denote as $\boldsymbol{E}_{n}^{(0)}$ and $\boldsymbol{B}_{n}^{(0)}$. That is, $\boldsymbol{E}_{n} \approx \boldsymbol{E}_{n}^{(0)}$ and $\boldsymbol{B}_{n} \approx \boldsymbol{B}_{n}^{(0)}$. The minimum corrective terms are related to $\boldsymbol{P}^{(0)}$ and $\boldsymbol{M}^{(0)}$, and satisfy the following equations,

$$
\begin{aligned}
& \nabla \cdot \boldsymbol{B}_{n}^{(0)}=0, \\
& \nabla \times \boldsymbol{E}_{n}^{(0)}+c^{-1} \partial_{t} \boldsymbol{B}_{n}^{(0)}=\mathbf{0}, \\
& \nabla \cdot \boldsymbol{E}_{n}^{(0)}=-\nabla \cdot \boldsymbol{P}^{(0)}, \\
& \nabla \times \boldsymbol{B}_{n}^{(0)}-c^{-1} \partial_{t} \boldsymbol{E}_{n}^{(0)}=c^{-1} \partial_{t} \boldsymbol{P}^{(0)}+\nabla \times \boldsymbol{M}^{(0)} .
\end{aligned}
$$

These are Maxwell's equations for $\boldsymbol{E}_{n}^{(0)}$ and $\boldsymbol{B}_{n}^{(0)}$. Although we use "minimum corrective terms" expressly, these terms are frequently considered in the literature $[11,24-26]$. As can be seen from equations (4) and (5), the linearization is done by replacing all $\boldsymbol{E}$ and $\boldsymbol{B}$ in $\boldsymbol{P}$ and $\boldsymbol{M}$ by the classical terms. On the other hand, there is another procedure of the linearization, i.e., one of each cubic term is replaced by the nonlinear correction term. A change of the refractive index [23] is a characteristic effect of the linearization, and note that it is beyond the scope of the minimum corrective terms. By eliminating $\boldsymbol{B}_{n}^{(0)}$, using

$$
\boldsymbol{S}^{(0)}=\nabla\left(\nabla \cdot \boldsymbol{P}^{(0)}\right)-c^{-2} \partial_{t}^{2} \boldsymbol{P}^{(0)}-c^{-1} \partial_{t} \nabla \times \boldsymbol{M}^{(0)},
$$

a wave equation for $\boldsymbol{E}_{n}^{(0)}$ is obtained as,

$$
\left(\triangle-c^{-2} \partial_{t}^{2}\right) \boldsymbol{E}_{n}^{(0)}=-\boldsymbol{S}^{(0)} .
$$

This wave equation cannot be treated alone because its solution can violate the third equation in equation (5). However, this wave equation is suggestive and will be a guide of this study. We can expect that a sufficient condition that the minimum corrective terms increase spatially or temporally will be given by

$$
\boldsymbol{S}^{(0)} \neq \mathbf{0} \text { and }\left(\triangle-c^{-2} \partial_{t}^{2}\right) \boldsymbol{S}^{(0)}=\mathbf{0} .
$$

Such a non-zero source term $\boldsymbol{S}^{(0)}$ itself is a solution of the wave equation. This means that the phases, or the cycles, of the source and the minimum corrective electric field can match locally, and therefore, the source can always operate to increase the amplitude of the corrective electric field. If the source term satisfies above conditions, it can act as the resonant external force, in the sense of classical mechanics. We use the term "resonance" in this context. Note that this word is used only within the range of minimum corrective terms. Then the main purposes of this study are constructing such classical terms, confirming that $\boldsymbol{E}_{n}^{(0)}$ and $\boldsymbol{B}_{n}^{(0)}$ surely increase resonantly, and evaluating the applicable range of this approximation. In the following, we see several examples. However, note that the choice of the classical term is not essential. It is also possible to consider more complicated form to reproduce an experimental situation and also in this case, the main problem is the conditions in equation (8).

\section{Stationary minimum corrections}

First, we consider stationary classical terms and the corresponding minimum corrective terms through a stationary special solution of equation (5). The special solution is suitable to study an effect of nonlinear Lagrangian because it does not appear in classical electromagnetism.

A simple classical term is a plane wave. One mode is characterized by $(\omega, \boldsymbol{k})$, where $\omega$ is the frequency, $\boldsymbol{k}$ is the wave vector, and they satisfy $\omega=c|\boldsymbol{k}| \neq 0$. Using constant vectors $\boldsymbol{E}_{p}$ and $\boldsymbol{B}_{p}$, the electric field and magnetic flux density are given by $\boldsymbol{E}_{p} \exp [i(\boldsymbol{k} \cdot \boldsymbol{x}-\omega t)]$ and $\boldsymbol{B}_{p} \exp [i(\boldsymbol{k}$. $\boldsymbol{x}-\omega t)]$, respectively. They satisfy $\boldsymbol{k} \cdot \boldsymbol{E}_{p}=0$ and $\boldsymbol{B}_{p}=$ $\hat{\boldsymbol{k}} \times \boldsymbol{E}_{p}$, where $\hat{\boldsymbol{k}}=\boldsymbol{k} /|\boldsymbol{k}|$.

Because $\boldsymbol{P}^{(0)}$ and $\boldsymbol{M}^{(0)}$ are cubic with respect to the classical terms, we consider three modes, i.e., $\left(\omega_{1}, \boldsymbol{k}_{1}\right),\left(\omega_{2}, \boldsymbol{k}_{2}\right)$, and $\left(\omega_{3}, \boldsymbol{k}_{3}\right)$. Let $\boldsymbol{K}=\boldsymbol{k}_{1}+\boldsymbol{k}_{2}+\boldsymbol{k}_{3}$ and $\Omega=\omega_{1}+\omega_{2}+\omega_{3}$. In the case of $|\boldsymbol{K}| \neq \Omega / c$, a stationary special solution of equation (5) is proportional to $\exp [i(\boldsymbol{K} \cdot \boldsymbol{x}-\Omega t)]$ and it is bounded in the entire space and time. In the case of $|\boldsymbol{K}|=\Omega / c$, the right-hand sides of the third and fourth equations in equation (5) become 
zero and a stationary special solution is also zero. Because the special solution of $\boldsymbol{E}_{n}^{(0)}$ is bounded, the only method to enlarge it is to enlarge the classical term, as is required in many previous studies $[11,27]$.

In the next case, the classical term is composed of a plane wave and constant fields. Each term of $\boldsymbol{P}^{(0)}, \boldsymbol{M}^{(0)}$, and $\boldsymbol{S}^{(0)}$ can be classified into the following four types. In the first type, all three of the electric field and magnetic flux density are those of the plane wave. In the second type, two are the plane wave and one is the constant fields. In the third type, one is the plane wave and two are the constant fields. In the fourth type, all three are the constant fields. For the first type, we already stated that a stationary special solution is bounded. The same result is obtained for the second type. In addition, the minimum corrective terms are clearly zero for the fourth type.

We then calculate for the remaining third type. We express a plane wave by $\boldsymbol{E}_{p} \exp [i(\boldsymbol{k} \cdot \boldsymbol{x}-\boldsymbol{\omega} t)]$ and $\boldsymbol{B}_{p} \exp [i(\boldsymbol{k} \cdot \boldsymbol{x}-\omega t)]$. The constant electric field and magnetic flux density are independently given by $\boldsymbol{E}_{s}$ and $\boldsymbol{B}_{s}$, respectively. Let $F_{s}=E_{s}^{2}-B_{s}^{2}$ and $G_{s}=\boldsymbol{E}_{s} \cdot \boldsymbol{B}_{s}$. Defining

$$
\begin{aligned}
\tilde{\boldsymbol{P}}_{0}= & 8 C_{2,0}\left(\boldsymbol{E}_{p} \cdot \boldsymbol{E}_{s}-\boldsymbol{B}_{p} \cdot \boldsymbol{B}_{s}\right) \boldsymbol{E}_{s} \\
& +2 C_{0,2}\left(\boldsymbol{E}_{p} \cdot \boldsymbol{B}_{s}+\boldsymbol{E}_{s} \cdot \boldsymbol{B}_{p}\right) \boldsymbol{B}_{s},
\end{aligned}
$$

we obtain $\boldsymbol{P}^{(0)}=\boldsymbol{P}_{0} \exp [i(\boldsymbol{k} \cdot \boldsymbol{x}-\omega t)]$, where $\boldsymbol{P}_{0}=$ $4 C_{2,0} F_{s} \boldsymbol{E}_{p}+2 C_{0,2} G_{s} \boldsymbol{B}_{p}+\tilde{\boldsymbol{P}}_{0}$. Similarly, defining

$$
\begin{aligned}
\tilde{\boldsymbol{M}}_{0}= & -8 C_{2,0}\left(\boldsymbol{E}_{p} \cdot \boldsymbol{E}_{s}-\boldsymbol{B}_{p} \cdot \boldsymbol{B}_{s}\right) \boldsymbol{B}_{s} \\
& +2 C_{0,2}\left(\boldsymbol{E}_{p} \cdot \boldsymbol{B}_{s}+\boldsymbol{E}_{s} \cdot \boldsymbol{B}_{p}\right) \boldsymbol{E}_{s}
\end{aligned}
$$

we obtain $\boldsymbol{M}^{(0)}=\boldsymbol{M}_{0} \exp [i(\boldsymbol{k} \cdot \boldsymbol{x}-\omega t)]$, where $\boldsymbol{M}_{0}=$ $-4 C_{2,0} F_{s} \boldsymbol{B}_{p}+2 C_{0,2} G_{s} \boldsymbol{E}_{p}+\tilde{\boldsymbol{M}}_{0}$.

In $\boldsymbol{P}^{(0)}$ and $\boldsymbol{M}^{(0)}$, the terms that contain $F_{s}$ or $G_{s}$ do not work as a source in equation (5). Therefore, they make no contribution for the minimum corrective terms. On the other hand, $\tilde{\boldsymbol{P}}_{0}$ and $\tilde{\boldsymbol{M}}_{0}$ can be sources and we obtain

$$
\boldsymbol{S}^{(0)}=k^{2}\left[\tilde{\boldsymbol{P}}_{0}-\hat{\boldsymbol{k}}\left(\hat{\boldsymbol{k}} \cdot \tilde{\boldsymbol{P}}_{0}\right)-\hat{\boldsymbol{k}} \times \tilde{\boldsymbol{M}}_{0}\right] e^{i(\boldsymbol{k} \cdot \boldsymbol{x}-\omega t)} .
$$

By appropriately choosing the constant fields, this satisfies equation (8). We finally obtain a stationary special solution as

$$
\begin{aligned}
\boldsymbol{E}_{n}^{(0)}= & \left\{\frac{i \boldsymbol{k} \cdot \boldsymbol{x}}{2}\left[\tilde{\boldsymbol{P}}_{0}-\hat{\boldsymbol{k}}\left(\hat{\boldsymbol{k}} \cdot \tilde{\boldsymbol{P}}_{0}\right)-\hat{\boldsymbol{k}} \times \tilde{\boldsymbol{M}}_{0}\right]\right. \\
& \left.-\hat{\boldsymbol{k}}\left(\hat{\boldsymbol{k}} \cdot \tilde{\boldsymbol{P}}_{0}\right)\right\} e^{i(\boldsymbol{k} \cdot \boldsymbol{x}-\omega t)} \\
\boldsymbol{B}_{n}^{(0)}= & \frac{1+i \boldsymbol{k} \cdot \boldsymbol{x}}{2} \hat{\boldsymbol{k}} \times\left(\tilde{\boldsymbol{P}}_{0}-\hat{\boldsymbol{k}} \times \tilde{\boldsymbol{M}}_{0}\right) e^{i(\boldsymbol{k} \cdot \boldsymbol{x}-\omega t)} .
\end{aligned}
$$

As is expected, $\boldsymbol{E}_{n}^{(0)}$ increases with distance provided that the direction is not orthogonal to $\boldsymbol{k}$ and the quantity within the square brackets is not zero. Concurrently, $\boldsymbol{B}_{n}^{(0)}$ increases with distance. Note that the same square brackets appear in equation (11). While the minimum corrective terms are good approximation, the electric field and magnetic flux density can increase. Naturally, this solution does not directly assert that the nonlinear correction diverges. If the minimum corrective terms become sufficiently large, we must then consider the terms that were initially ignored, such as another linearization. Thus, the divergence will be restricted. In addition, note that equation (12) is just a special solution of the wave equation in equation (7). The solution for a physical problem is obtained by solving with appropriate initial and boundary conditions. The homogeneous solution is specified by the initial and boundary conditions, and the solution is the sum of the homogeneous solution and the special solution. Therefore, whether the resonant enhancement is possible must be discussed by including the homogeneous solution. However, the homogeneous solution never becomes the same function as the special solution and therefore, the resonant enhancement appears in the special solution may not be cancelled.

For the moment, let us see the special solution in equation (12) in detail. Firstly, we evaluate the applicable limit of distance where the minimum corrective terms are good approximation. For simplicity, we suppose $E_{s}=0, E_{p}=$ $B_{p} \ll B_{s}$, the plane wave propagates to the $x$ direction, and $\boldsymbol{E}_{p}$ and $\boldsymbol{B}_{s}$ have only the $y$ component. In this case, only the $y$ component is nonzero for $\boldsymbol{E}_{n}^{(0)}$ and it is given by $E_{n y}^{(0)}=i k x C_{0,2} E_{p} B_{s}^{2} \exp [i(k x-\omega t)]$. The minimum corrective terms must be much smaller than the classical terms. It can be expressed as $k|x| C_{0,2} E_{p} B_{s}^{2} \ll E_{p}$ and converting to SI units, we obtain

$$
|x| \ll \frac{\lambda}{2 \pi C_{0,2} \mu_{0}^{-1} B_{s}^{2}},
$$

where $\lambda=2 \pi / k$ is the wavelength. If a plane wave and a constant magnetic flux density are given in a distance expressed in the right-hand side, a resonantly increased corrective electric field will be observed. Provided that $k|x| \gg 1$, the same condition is derived from the magnetic flux density.

For example, let us evaluate for $B_{s}=1(\mathrm{~T})$ and $\lambda=400(\mathrm{~nm})$, and supposing the above calculation is valid while $\left|\boldsymbol{E}_{n}^{(0)}\right|$ increases to $E_{p} / 10$. If we denote $x_{0.1}$ as the necessary distance for the resonant increase, then $x_{0.1} \approx 6.86 \times 10^{14}(\mathrm{~m})$. Imposing in this distance is not experimentally realistic. Thus, even if equation (12) is a part of a physical solution with appropriate initial and boundary conditions, the resonant effect will not be detectable in an experimental length scale.

The calculation so far is limited in the applicable range of the linearization of the minimum corrective terms. The plane wave has a classical dispersion relation and the resonant solution in equation (12) is obtained. Beyond this approximation, the plane wave in the static magnetic flux density is no longer classical, i.e., its refractive index can differ from unity, as can be seen from another linearization. However, it does not lose the essence of the resonance. For example, the resonance can still be expected in the following system. A static magnetic flux density has only the $y$ component and exists only in $x \geq 0$. The incident light propagates along the $x$ axis and it is a classical plane wave in $x<0$. In $x \geq 0$, it will eventually 
be modulated by the nonlinear effect of the magnetic flux density. However, at sufficiently small positive $x$, such a modulation is negligibly small, and therefore, the source term $\boldsymbol{S}^{(0)}$ will approximately satisfy equation (8) and the minimum corrective terms behave as resonant.

Within the approximation of the minimum corrective term, the essence of the resonance is an existence of $\boldsymbol{S}^{(0)}$ such that it satisfies equation (8). Equation (12) is a concise demonstration. However, for more precise calculation, the combination of a static magnetic flux density and a plane wave will require a discussion beyond the approximation and it makes the essence of this study obscure.

Then, in the next section, we consider another system where the static magnetic flux density and plane wave in the entire space do not appear; besides, the approximation of the minimum corrective term is assured.

\section{Minimum corrections in one-dimensional cavity}

In addition to the above reason, to demonstrate that the resonance is not only a mathematical solution but also a physical solution, we herein consider a one-dimensional cavity with appropriate initial and boundary conditions. In the cavity, the classical term is not a plane wave but a standing wave. Hence, $F$ is not identically zero, and as we show, the resonance can take place without a constant field.

Two mirrors are located at $x=0$ and $x=l$. The interior of the cavity is $0 \leq x \leq l$. All functions depend only on $x$ and $t$. Let $k$ be the wavenumber and we suppose $k l=\pi n$ for a certain natural number $n$. The frequency is given by $\omega=c k$. The unit vectors of the $y$ and $z$ directions are expressed by $\boldsymbol{e}_{y}$ and $\boldsymbol{e}_{z}$, respectively.

With an amplitude $A$, the standing electric field and magnetic flux density are given by

$$
\boldsymbol{E}_{c}=A \sin k x \sin \omega t \boldsymbol{e}_{y}, \quad \boldsymbol{B}_{c}=A \cos k x \cos \omega t \boldsymbol{e}_{z} .
$$

Although these are classical standing waves, they do not satisfy the nonlinear Maxwell's equations in equation (3). Thusly, the nonlinear corrections appear. We obtain

$$
\begin{gathered}
\boldsymbol{S}^{(0)}=2 k^{2} C_{2,0} A^{3}(\sin 3 k x \sin \omega t-3 \sin k x \sin 3 \omega t \\
+2 \sin k x \sin \omega t) \boldsymbol{e}_{y} .
\end{gathered}
$$

Note that the third term in the parentheses of the righthand side satisfies equation (8).

We give the initial and boundary conditions on the minimum corrective terms. At the initial time $t=0$, they must be much smaller than $A$. Then, we suppose the initial distributions as $\boldsymbol{E}_{n}^{(0)}(x, 0)=\mathbf{0}$ and $\boldsymbol{B}_{n}^{(0)}(x, 0)=\mathbf{0}$. The boundary conditions at $x=0$ and $x=l$ depend on the property of the mirrors. For simplicity, we suppose that the mirrors are perfect conductors. In this case, the components of the electric field parallel to the mirror surface must be zero. Similarly, the component of the magnetic flux density vertical to the mirror surface must be zero. Therefore, the boundary conditions are given by
$E_{n y}^{(0)}(0, t)=0, E_{n y}^{(0)}(l, t)=0, E_{n z}^{(0)}(0, t)=0, E_{n z}^{(0)}(l, t)=$ $0, B_{n x}^{(0)}(0, t)=0$, and $B_{n x}^{(0)}(l, t)=0$. These standard initial and boundary conditions are sufficient for the unique solution [28]. Finally, we obtain as,

$$
\begin{aligned}
\boldsymbol{E}_{n}^{(0)}(x, t)= & C_{2,0} A^{3}\left\{\sin k x\left[\frac{3}{4}(\sin 3 \omega t-3 \sin \omega t)-2 \omega t \cos \omega t\right]\right. \\
& \left.+\frac{1}{4} \sin 3 k x(\sin \omega t-3 \sin 3 \omega t)\right\} \boldsymbol{e}_{y}, \\
\boldsymbol{B}_{n}^{(0)}(x, t)= & C_{2,0} A^{3}\left\{\cos k x\left[\frac{1}{4}(\cos 3 \omega t-\cos \omega t)+2 \omega t \sin \omega t\right]\right. \\
& \left.+\frac{3}{4} \cos 3 k x(\cos \omega t-\cos 3 \omega t)\right\} \boldsymbol{e}_{z} .
\end{aligned}
$$

Note that $\omega t \cos \omega t$ appears in $\boldsymbol{E}_{n}^{(0)}$ and $\omega t \sin \omega t$ appears in $\boldsymbol{B}_{n}^{(0)}$. As we expected, the resonant effect is represented as a temporal function. The amplitudes of both $\boldsymbol{E}_{n}^{(0)}$ and $\boldsymbol{B}_{n}^{(0)}$ increase with time. Although this equation may suggest that the energy increases infinitely, it is not correct. While the minimum corrective terms are good approximations, their energies are just a small fraction of the total energy. By considering the entire $\boldsymbol{P}, \boldsymbol{M}$, and nonlinear corrections, the total energy is conserved.

We next evaluate the time when the minimum corrective terms can be a good approximation, i.e., both $\left|\boldsymbol{E}_{n}^{(0)}\right|$ and $\left|\boldsymbol{B}_{n}^{(0)}\right|$ are much smaller than $A$. It can be given by $2 C_{2,0} A^{3} \omega t \ll A$, i.e.,

$$
t \ll \frac{1}{2 C_{2,0} A^{2} \omega} .
$$

In this condition, a derivative of the minimum corrective terms is much smaller than the magnitude of the corresponding derivative of the classical terms, e.g., $\left|\partial_{t} \boldsymbol{E}_{n}^{(0)}\right| \ll \omega A$. Therefore, the above calculation may be a good approximation. The right-hand side of equation (17) serves as a threshold of the applicable limit and a guide for the necessary time to sufficiently increase the minimum corrective terms. A large resonant effect will be more rapidly realized by shortening this value. If the necessary time is shortened, the number of reflection decreases and unwanted effects such as mirror heating or energy loss by mirror surfaces will be restricted.

For example, we evaluate by supposing the above calculation is valid for $\left|\boldsymbol{E}_{n}^{(0)}\right| \leq A / 10$. For converting to SI units, $A^{2}$ is replaced by $\varepsilon_{0} E_{a}^{2}$, where $E_{a}$ is the amplitude of the standing electric field. Because a standing wave is frequently used in modern experiments of optical lattice [29], we evaluate the applicable limit by using the reference. A typical wavelength and intensity can be $\lambda=400(\mathrm{~nm})$ and $c \varepsilon_{0} E_{a}^{2} \approx 10^{10}\left(\mathrm{~W} / \mathrm{m}^{2}\right)$, respectively. In this case, the evaluated time can exceed a thousand year. This value is understandable because the resonant effect has not been reported in such experiments. To observe the resonance, an experimental set up will be optimized for this purpose depending on its advantage. For example, if a sensitive measurement is possible, a short time resonance may be sufficient. On the other hand, if the system can be kept for a long time, the sensitive measurement may not be 
required, rather, it is sufficient to wait for the nonlinear correction to become large.

A more rigorous and realistic calculation will be required for the design of an experimental setup. For example, all the initial values are zero and the input to generate the classical standing wave is given as the boundary conditions at the mirror surface, not as in equation (14). Provided that the input is completed in sufficiently short time, a similar result will be obtained and equation (17) can be used as the applicable limit.

In an experimental viewpoint, the generation and maintenance of a standing wave will become difficult as intensity increases. On the other hand, the value of the righthand side of equation (17) becomes too large for a small intensity. In such a case, a strong constant magnetic flux density would be used for the shortening. If there is an external constant magnetic flux density $\boldsymbol{B}_{s}=B_{s} \boldsymbol{e}_{z}$, the amplitude of the resonant part of the minimum corrective electric field is given by $2 C_{2,0} A\left(A^{2}+2 B_{s}^{2}\right) \omega t$. Therefore, converting to SI units, the applicable limit in this case is evaluated as

$$
t \ll \frac{1}{2 C_{2,0}\left(\varepsilon_{0} E_{a}^{2}+2 \mu_{0}^{-1} B_{s}^{2}\right) \omega} .
$$

As a value of strong static magnetic flux density, we use $B_{s}=17.6(\mathrm{~T})[30,31]$. Substituting this magnetic flux density and the electric field corresponds to the intensity of $10^{10}\left(\mathrm{~W} / \mathrm{m}^{2}\right)$ into the right-hand side of equation (18), the evaluated applicable limit is about $1.3 \times 10^{5}$ (s). According to reference [30], the magnetic flux density can be retained about 10 min which is within the applicable limit. Then, at $t=600(\mathrm{~s})$, the ratio of the amplitude of the resonance to the classical standing wave is $2 C_{2,0}\left(\varepsilon_{0} E_{a}^{2}+2 \mu_{0}^{-1} B_{s}^{2}\right) \omega t \approx 4.6 \times 10^{-3}$. This ratio would be distinguishable by a current sensitive measurement. It will be promising to evaluate the resonant effect by considering more realistic conditions for an experimental detection.

It may be worth referring to the pulsed magnetic flux density. The pulsed magnetic flux density can exceed $10^{2}(\mathrm{~T})[32]$ and $10^{3}(\mathrm{~T})$ [33]. These systems should not be considered as static, while the effect of such strong fields will be valuable.

\section{Final remarks}

In nonlinear Maxwell's equations, an electromagnetic wave itself can be a source for another electromagnetic wave. We have utilized this property and shown that a classical electromagnetic wave can be a resonant source for the nonlinear corrective terms. The essence is to construct the source term $\boldsymbol{S}^{(0)}$ satisfying equation (8). For a stationary classical term, it was shown that a special solution can increase with distance. Within the applicable range of the minimum corrective terms, a nonzero value of the square brackets in equation (11) is a sufficient condition for the resonance. The resonant effect can also be realized with time, such as shown in a one-dimensional cavity.
The minimum corrective terms are solved with the initial and boundary conditions and given by equation (16). To obtain the solution, we have set the initial distributions as zero. Although they must be much smaller than the amplitude of the classical term, they may not be zero. For any initial distributions that are compatible with the boundary conditions, equation (5) has the unique solution and it also shows the resonant enhancement. In particular, the applicable limit can be given as the same condition as equation (17).

Throughout the study, we have used the word "resonance". In general, a resonance can be measured by the quality factor. To calculate the value in the present study, we need to evaluate the energy flow from the classical term to the nonlinear corrective term. For example, in the cavity system, such a calculation requires much longer time than the applicable limit shown in equation (17). Therefore, calculating the quality factor cannot be completed within the present approximation. In such a long time scale, the corrective term can be comparably large and the energy will flow bidirectionally between the classical and corrective terms. Thus, "resonance" is not always suitable. Therefore, we have limited to use within the present approximation. For example, "resonance in a short range" will be more precise to describe the phenomenon.

The present study can be regarded as a generalization of a resonance once investigated [24]. An important difference is that we have elucidated that the resonance is intrinsic to nonlinear Maxwell's equations. The resonance does not necessarily require a specific physical configuration, as we have shown for the classical term that consists of a plane wave and constant fields. Although we have also calculated for a cavity system, it was only to illustrate an example of a closed light path and not essential to the resonance. A similar resonance can be easily found in a two-dimensional cavity.

We have already mentioned that the resonance does not continue infinitely and evaluated the applicable limit. Beyond the applicable limit is also of interest because the nonlinearity directly appears [34-37]. In particular, different types of resonance can be expected in reference [38] and in a self-focusing (an analogy will be found in Ref. [39]).

In the present study, the nonlinear Lagrangian is limited to the quadratic form with respect to $F$ and $G$. Therefore, the resonant effect can be utilized to detect the nonlinear optical effect of vacuum but may not directly provoke the pair production. However, if a similar calculation can be done in the Lagrangian including higher order terms, unexpected effects can be found.

Because of the resonance, the nonlinear corrective terms can increase with long distance or long time. This feature is remarkably different from many considered nonlinear optical effects where the maximum effect is limited by the magnitude of the input classical terms. Considering the origin and simplicity of the resonance, the conditions and results shown in this study can be combined with existing experimental proposals and shall contribute to realistic experimental detection of nonlinear vacuum. 
The author thanks Dr. M. Nakai, Dr. K. Mima, and Mr. T. Hara for the discussions on the resonance and its application, and to Dr. N. Sarukura for the experimental feasibility of a cavity. The author appreciates Dr. J. Gabayno for checking the logical consistency of the text.

Publisher's Note The EPJ Publishers remain neutral with regard to jurisdictional claims in published maps and institutional affiliations.

Open Access This is an open access article distributed under the terms of the Creative Commons Attribution License (https://creativecommons.org/licenses/by/4.0/), which permits unrestricted use, distribution, and reproduction in any medium, provided the original work is properly cited.

\section{References}

1. M. Born, L. Infeld, R.H. Fowler, Proc. R. Soc. London. Ser. A 144, 425 (1934)

2. W. Heisenberg, H. Euler, Z. Phys. 98, 714 (1936)

3. J. Schwinger, Phys. Rev. 82, 664 (1951)

4. I. Drebot, D. Micieli, E. Milotti, V. Petrillo, E. Tassi, L. Serafini, Phys. Rev. Accel. Beams 20, 043402 (2017)

5. L. Labun, J. Rafelski, Acta Phys. Pol. B 43, 2237 (2012)

6. J.S. Heyl, L. Hernquist, Astrophys. J. 618, 463 (2005)

7. R.P. Mignani, V. Testa, K. Wu, S. Zane, D. Gonzalez Caniulef, R. Turolla, R. Taverna, MNRAS 465, 492 (2016)

8. S.H. Mazharimousavi, M. Halilsoy, Found. Phys. 42, 524 (2012)

9. V.I. Denisov, N.V. Kravtsov, I.V. Krivchenkov, Opt. Spectrosc. 100, 641 (2006)

10. I. Turcu, F. Negoita, D. Jaroszynski, P. Mckenna, S. Balascuta, D. Ursescu, I. Dancus, M. Cernaianu, M. Tataru, P. Ghenuche, D. Stutman, A. Boianu, M. Risca, M. Toma, C. Petcu, G. Acbas, S. Yoffe, A. Noble, B. Ersfeld, N. Zamfir, Romanian Rep. Phys. 68, S145 (2016)

11. E. Lundström, G. Brodin, J. Lundin, M. Marklund, R. Bingham, J. Collier, J.T. Mendonça, P. Norreys, Phys. Rev. Lett. 96, 083602 (2006)

12. G.V. Dunne, Eur. Phys. J. D 55, 327 (2009)

13. F. Della Valle, E. Milotti, A. Ejlli, G. Messineo, L. Piemontese, G. Zavattini, U. Gastaldi, R. Pengo, G. Ruoso, Phys. Rev. D 90, 092003 (2014)

14. E. Milotti, F. Della Valle, G. Zavattini, G. Messineo, U. Gastaldi, R. Pengo, G. Ruoso, D. Babusci, C. Curceanu, M. Iliescu, C. Milardi, Int. J. Quantum Inf. 10, 1241002 (2012)
15. F. Briscese, Phys. Rev. A 96, 053801 (2017)

16. H. Gies, F. Karbstein, N. Seegert, New J. Phys. 15, 083002 (2013)

17. M. Marklund, P.K. Shukla, Rev. Mod. Phys. 78, 591 (2006)

18. M. Marklund, J. Lundin, Eur. Phys. J. D 55, 319 (2009)

19. B. King, T. Heinzl, High Power Laser Sci. Eng. 4, e5 (2016)

20. T. Inada, T. Yamazaki, T. Yamaji, Y. Seino, X. Fan, S. Kamioka, T. Namba, S. Asai, Appl. Sci. 7, 671 (2017)

21. A. Di Piazza, C. Müller, K.Z. Hatsagortsyan, C.H. Keitel, Rev. Mod. Phys. 84, 1177 (2012)

22. J. Plebanski, Lectures on non-linear electrodynamics: an extended version of lectures given at the Niels Bohr Institute and NORDITA, Copenhagen, in October 1968 (Copenhagen : NORDITA, 1970), https://www.osti. gov/servlets/purl/4071071

23. M. Fouché, R. Battesti, C. Rizzo, Phys. Rev. D 93, 093020 (2016)

24. G. Brodin, M. Marklund, L. Stenflo, Phys. Rev. Lett. 87, $171801(2001)$

25. D. Eriksson, G. Brodin, M. Marklund, L. Stenflo, Phys. Rev. A 70, 013808 (2004)

26. B. King, C.H. Keitel, New J. Phys. 14, 103002 (2012)

27. A. Fedotov, N. Narozhny, Phys. Lett. A 362, 1 (2007)

28. M. Ikawa, Introduction to Partial Differential Equation, 1st edn. (Shokabo Co., Ltd., Tokyo, 1996)

29. H. Katori, V.D. Ovsiannikov, S.I. Marmo, V.G. Palchikov, Phys. Rev. A 91, 052503 (2015)

30. J.H. Durrell, A.R. Dennis, J. Jaroszynski, M.D. Ainslie, K.G.B. Palmer, Y.H. Shi, A.M. Campbell, J. Hull, M. Strasik, E.E. Hellstrom, D.A. Cardwell, Supercond. Sci. Technol. 27, 082001 (2014)

31. J.H. Durrell, M.D. Ainslie, D. Zhou, P. Vanderbemden, T. Bradshaw, S. Speller, M. Filipenko, D.A. Cardwell, Supercond. Sci. Technol. 31, 103501 (2018)

32. M. Jaime, R. Daou, S.A. Crooker, F. Weickert, A. Uchida, A.E. Feiguin, C.D. Batista, H.A. Dabkowska, B.D. Gaulin, Proc. Natl. Acad. Sci. 109, 12404 (2012)

33. D. Nakamura, A. Ikeda, H. Sawabe, Y.H. Matsuda, S. Takeyama, Rev. Sci. Instrum. 89, 095106 (2018)

34. J. Mendonca, M. Marklund, P. Shukla, G. Brodin, Phys. Lett. A 359, 700 (2006)

35. L.M. Kovachev, D.A. Georgieva, K.L. Kovachev, Opt. Lett. 37, 4047 (2012)

36. H. Kadlecová, G. Korn, S.V. Bulanov, Phys. Rev. D 99, $036002(2019)$

37. S.V. Bulanov, P.V. Sasorov, F. Pegoraro, H. Kadlecová, S.S. Bulanov, T.Z. Esirkepov, N.N. Rosanov, G. Korn, Phys. Rev. D 101, 016016 (2020)

38. A. Arza, R.G. Elias, Phys. Rev. D 97, 096005 (2018)

39. B.I. Cohen, B.F. Lasinski, A.B. Langdon, J.C. Cummings, Phys. Fluids B: Plasma Phys. 3, 766 (1991) 